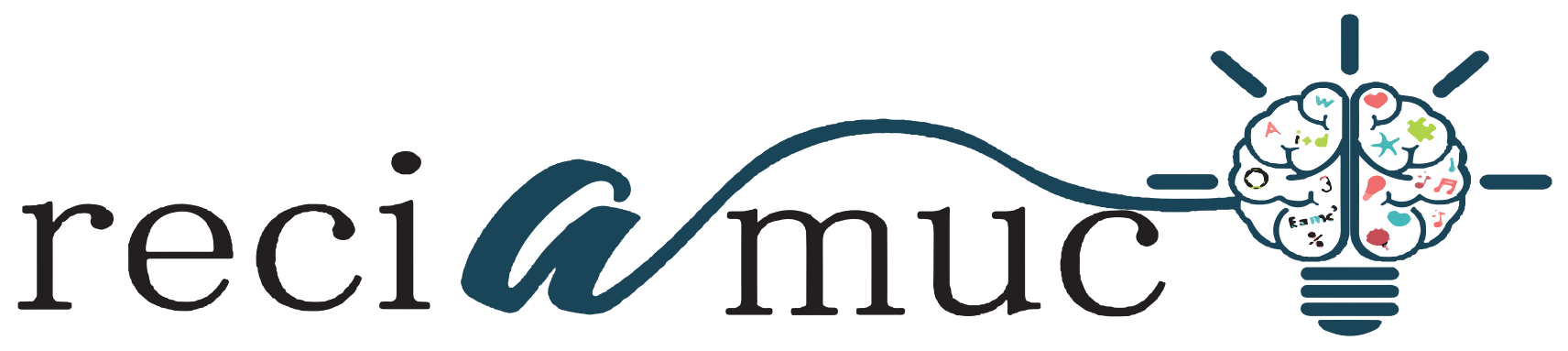

DOI: 10.26820/reciamuc/5.(1).ene.2021.357-367

URL: https://reciamuc.com/index.php/RECIAMUC/article/view/625

EDITORIAL: Saberes del Conocimiento

REVISTA: RECIAMUC

ISSN: 2588-0748

TIPO DE INVESTIGACIÓN: Artículo de revisión

Código UNESCO: 32 Ciencias Médicas

PAGINAS: 357-367

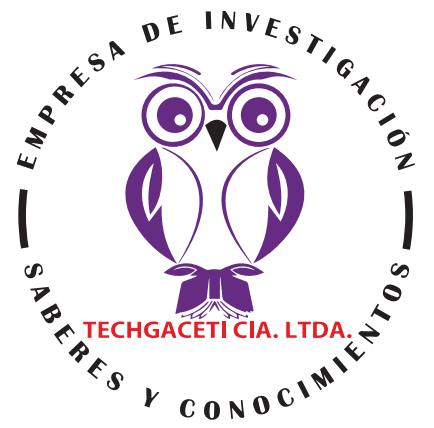

\title{
Utilidad de la ecografía torácica en pacientes con enfermedad pleuropulmonar
}

Usefulness of thoracic ultrasound in patients with pleuropulmonary disease

Utilidade do ultra-som torácico em pacientes com doença pleuropulmonar

\section{Evelyn Esthela Sánchez Riera'; Lucia Estefanía Osorio Vera²; Luis Enrique Quisiguiña Avellan³; Melissa Xiomara Herrera Vera ${ }^{4}$}

RECIBIDO: 10/11/2020 ACEPTADO: 29/11/2020 PUBLICADO: 31/01/2021

1. Médico de la Universidad de Guayaquil; Investigador Independiente; Guayaquil, Ecuador; evelyn.sanchez.riera@gmail.com; https://orcid.org/0000-0002-3897-9037

2. Médico de la Universidad de Guayaquil; Investigador Independiente; Guayaquil, Ecuador; luciaoso14@ hotmail.com; https://orcid.org/0000-0002-0193-0248

3. Médico de la Universidad Católica de Santiago de Guayaquil; Investigador Independiente; Guayaquil, Ecuador; luis-enrique2191@hotmail.com; https://orcid.org/0000-0002-7615-0676

4. Médico de la Universidad Católica de Santiago de Guayaquil; Investigador Independiente; Guayaquil, Ecuador; melissaherrera8810@gmail.com; https://orcid.org/0000-0001-5310-2164

CORRESPONDENCIA

Evelyn Esthela Sánchez Riera

evelyn.sanchez.riera@gmail.com

Guayaquil, Ecuador

๑ RECIAMUC; Editorial Saberes del Conocimiento, 2021 


\section{RESUMEN}

Aunque se han realizado numerosos estudios sobre el uso de la ecografía para el examen de las estructuras torácicas, este procedimiento no es tan aceptado como la ecografía abdominal. La ecografía torácica comúnmente es utilizada en el diagnostico de parénquima pulmonar periférico, pleura, pared torácica, diafragma y mediastino, y también es de gran utilidad como guía en procedimientos intervencionistas diagnósticos y terapéuticos. Los escáneres portátiles más nuevos se pueden utilizar junto a la cama para detectar neoplasias y derrames pleurales, así como nódulos pulmonares periféricos del pulmón, incluso en pacientes gravemente enfermos. El engrosamiento focal de la pleura se puede detectar fácilmente con ecografía y se puede investigar más a fondo con una biopsia guiada por ecografía. La guía ecográfica también es recomendada durante el drenaje percutáneo del derrame pleural o la biopsia transtorácica de lesiones pulmonares periféricas, reduciendo así la incidencia de neumotórax relacionado con el procedimiento a casi cero. Por medio de la siguiente revisión literaria, se brinda información importante y de interés acerca de la ecografía torácica sus ventajas y el papel que juega en enfermedades pulmonares pleurales y periféricas, a través de la experiencia clínica en grupos de pacientes que presentan este tipo de patologías.

Palabras clave: Ecografía; pecho; pleura; pulmón; biopsia.

\section{ABSTRACT}

Although there have been numerous studies on the use of ultrasound to examine the thoracic structures, this procedure is not as widely accepted as abdominal ultrasound. Thoracic ultrasound is commonly used in the diagnosis of peripheral lung parenchyma, pleura, chest wall, diaphragm and mediastinum, and is also very useful as a guide in diagnostic and therapeutic interventional procedures. Newer portable scanners can be used at the bedside to detect pleural neoplasms and effusions, as well as peripheral lung nodules, even in seriously ill patients. Focal thickening of the pleura can be easily detected with ultrasound and can be further investigated with an ultrasound-guided biopsy. Ultrasound guidance is also recommended during percutaneous drainage of pleural effusion or transthoracic biopsy of peripheral lung lesions, thus reducing the incidence of procedure-related pneumothorax to almost zero. Through the following literary review, important and interesting information is provided about thoracic ultrasound, its advantages and the role it plays in pleural and peripheral lung diseases, through clinical experience in groups of patients with this type of pathology.

KeyWords: Ultrasound; chest; pleura; lung; biopsy.

\section{RESUMO}

Embora tenha havido numerosos estudos sobre o uso do ultra-som para examinar as estruturas torácicas, este procedimento não é tão amplamente aceito como o ultra-som abdominal. A ultrassonografia torácica é comumente usada no diagnóstico de parênquima pulmonar periférico, pleura, parede torácica, diafragma e mediastino, e também é muito útil como guia em procedimentos de diagnóstico e intervenção terapêutica. Novos scanners portáteis podem ser usados na beira do leito para detectar neoplasias pleurais e derrames, bem como nódulos pulmonares periféricos, mesmo em pacientes gravemente enfermos. O espessamento focal da pleura pode ser facilmente detectado com ultra-som e pode ser investigado posteriormente com uma biópsia guiada por ultra-som. A orientação por ultra-som também é recomendada durante a drenagem percutânea de derrame pleural ou biópsia transtorácica de lesões pulmonares periféricas, reduzindo assim a incidência de pneumotórax relacionado ao procedimento para quase zero. Através da seguinte revisão literária, são fornecidas informações importantes e interessantes sobre o ultra-som torácico, suas vantagens e o papel que desempenha nas doenças pulmonares pleurais e periféricas, através da experiência clínica em grupos de pacientes com este tipo de patologia.

Palavras-chave: Ultra-som; tórax; pleura; pulmão; biópsia. 


\section{Introducción}

La ecografía se puede utilizar para explorar las superficies de los pulmones a través de los espacios intercostales, pero la presencia de las costillas y de aire en el pulmón expandido reduce el valor de esta modalidad de imagen en el examen de estructuras torácicas más profundas.

Sin embargo, la ecografía se considera un método de diagnóstico confiable, económico, seguro y reproducible para el diagnóstico de pacientes con enfermedades del diafragma (neoplasias, paresia), pared torácica (abscesos, fístulas, neoplasias), pulmón (atelectasia, consolidación pulmonar), mediastino anterosuperior (neoplasias, linfomas, quistes), la región entre el tórax y el abdomen y, sobre todo, la pleura (masas extrapleurales, derrames pleurales) (Alcazar \& Arnedillo, 2016).

Gracias a la reciente difusión de sofisticados escáneres de ecografía equipados con tecnología Doppler de color y potencia y transductores especiales para exámenes transesofágicos y endobronquiales, la ecografía ahora se puede utilizar para investigar trastornos que involucran el esófago, los bronquios, los vasos sanguíneos bronquiales, el mediastino y los vasos del corazón. Aunque la tomografía computarizada sigue siendo el método de imagen de elección para el diagnóstico de estas afecciones, la ecografía torácica se puede considerar una herramienta complementaria importante en este contexto.

Hoy en día, la ecografía torácica se utiliza principalmente para guiar la biopsia transtorácica de lesiones pulmonares periféricas y el drenaje de derrames pleurales. "El uso cada vez más generalizado de agentes de contraste para ultrasonidos de segunda generación está ampliando aún más el papel de la ecografía torácica y está produciendo resultados prometedores en la caracterización de masas pulmonares periféricas" (Bokor, Chambers, \& Rees, 2011).
El examen ecográfico torácico se puede realizar junto a la cama y no se necesita una preparación específica del paciente. Sin embargo, los pacientes con insuficiencia respiratoria deben recibir terapia con medicamentos y oxígeno para aliviar sus síntomas durante el examen y reducir el riesgo de artefactos relacionados con el movimiento causado por la dificultad para respirar.

Las exploraciones de la pleura basal y el diafragma deben obtenerse con el paciente sentado y luego (cuando sea posible) en decúbito supino. En casos raros, se le puede pedir al paciente que se ponga de pie durante el examen (p. Ej., Cuando se explora el receso costofrénico en busca de un posible derrame) (Sperandeo, Caturelli, Sperandeo, \& Camagna, 2012).

Se pueden utilizar varios planos de exploración: "exploraciones intercostales, longitudinales, transversales y paravertebrales para explorar la pared torácica posterior, mientras que la pared anterior se suele investigar con intercostal, longitudinal, supra y paraesternal, subxifoidea y exploraciones supraclaviculares (El Fortia, El Gatit, \& Bendaoud, 2016). Se aprovechan ventanas acústicas específicas para mejorar la visualización de las estructuras examinadas. "En el lado derecho del cuerpo, el hígado proporciona una buena ventana para observar la pleura basal y la cúpula diafragmática (el paciente debe colocarse en posición supina y se le debe indicar que inhale profundamente)" (Lichtenstein, Meziere, Biderman, \& Gepner, 2011). El bazo proporciona una ventana similar para examinar las estructuras del lado izquierdo del tórax.

En tal sentido y de acuerdo a la importancia en la valoración de las enfermedades pleurales y pulmonares se presenta una revisión bibliográfica que ofrece información acerca del papel que juega la ecografía torácica en este tipo de patologías. 


\section{Metodología}

Para el desarrollo de este proceso investigativo, se plantea como metodología la encaminada hacia una orientación científica particular que se encuentra determinada por la necesidad de indagar en forma precisa y coherente una situación, en tal sentido Davila, (2015) define la metodología "como aquellos pasos anteriores que son seleccionados por el investigador para lograr resultados favorables que le ayuden a plantear nuevas ideas" (p.66)

Lo citado por el autor, lleva a entender que el desarrollo de la acción investigativa busca simplemente coordinar acciones enmarcadas en una revisión bibliográfica con el fin de complementar ideas previas relacionadas Papel de la ecografía torácica en la valoración de enfermedades pleurales y pulmonares a través de una revisión de literatura, para así finalmente elaborar un cuerpo de consideraciones generales que ayuden a ampliar el interés propuesto.

\section{Tipo de Investigación}

Dentro de toda práctica investigativa, se precisan acciones de carácter metodológico mediante las cuales se logra conocer y proyectar los eventos posibles que la determinan. En este sentido, la presente investigación corresponde al tipo documental, definido por Castro (2016), "se ocupa del estudio de problemas planteados a nivel teórico, la información requerida para abordarlos se encuentra básicamente en materiales impresos, audiovisuales y / o electrónicos". (p.41).

En consideración a esta definición, la orientación metodológica incluye la oportunidad de cumplir con una serie de actividades inherentes a la revisión y lectura de diversos documentos, donde se encuentran ideas explicitas relacionadas con los tópicos encargados de identificar una característica inmersa en el estudio. Por lo tanto, se realizaron continuas interpretaciones con el claro propósito de revisar aquellas aprecia- ciones propuestas por diferentes investigadores en relación al tema de interés, para luego dar la respectiva argumentación a los planteamientos, en función a las necesidades encontradas en la investigación, apoyados en las herramientas tecnológicas para la búsqueda de trabajos con valor científico disponibles en la web que tenían conexión con el objetivo principal de la investigación.

\section{Fuentes Documentales}

El análisis correspondiente a las características que predomina en el tema seleccionado, llevan a incluir diferentes fuentes documentales encargadas de darle el respectivo valor científico y en ese sentido cumplir con la valoración de los hechos a fin de generar nuevos criterios que sirven de referencia a otros procesos investigativos. Para Castro,(2016) las fuentes documentales incorporadas en la investigación documental o bibliográfica, "representa la suma de materiales sistemáticos que son revisados en forma rigurosa y profunda para llegar a un análisis del fenómeno" (p.41). Por lo tanto, se procedió a cumplir con la lectura previa determinada para encontrar aquellos aspectos estrechamente vinculados con el tema, con el fin de explicar mediante un desarrollo las respectivas apreciaciones generales de importancia.

\section{Técnicas para la Recolección de la Infor- mación}

La conducción de la investigación para ser realizada en función a las particularidades que determinan a los estudios documentales, tiene como fin el desarrollo de un conjunto de acciones encargadas de llevar a la selección de técnicas estrechamente vinculadas con las características del estudio. Bolívar, (2015), refiere, que es "una técnica particular para aportar ayuda a los procedimientos de selección de las ideas primarias y secundarias". (p.71).

Tal como lo expresa, Bolívar, (2015) "Las técnicas documentales proporcionan las herramientas esenciales y determinantes 
para responder a los objetivos formulados y llegar a resultados efectivos" (p. 58). Es decir, para responder con eficiencia a las necesidades investigativas, se introdujeron como técnica de recolección el método inductivo, que hizo posible llevar a cabo una valoración de los hechos de forma particular para llegar a la explicación desde una visión general. El autor Bolívar, (2015) tambien expresa que las técnicas de procesamiento de datos en los estudios documentales "son las encargadas de ofrecer al investigador la visión o pasos que deben cumplir durante su ejercicio, cada una de ellas debe estar en correspondencia con el nivel a emplear" (p. 123). Esto indica, que para llevar a cabo el procesamiento de los datos obtenidos una vez aplicadas las técnicas seleccionadas, tales como: fichas de resumen, textual, registros descriptivos entre otros, los mismos se deben ajustar al nivel que ha sido seleccionado.

\section{Resultados}

\section{Aplicaciones clínicas}

Las estructuras torácicas que pueden ser exploradas por ecografía incluyen; la superficie, 1-piel, 2-dermis, 3-músculos intercostales y membrana endo torácica, 4-grasa extra pleural y las pleuras parietal y visceral tal se observa en la (Figura 1). Una vez que el haz de ecografía ha penetrado en la pleura visceral, el aire de los pulmones lo dispersa por completo. "La impedancia acústica elevada generada en la interfaz entre los tejidos blandos superficiales y el aire en el pulmón da como resultado una línea ecogénica delgada ( $<3 \mathrm{~mm}$ ) conocida como línea pleural" (Targhetta, Bourgeois, Chavagneux, \& Balmes, 2002).

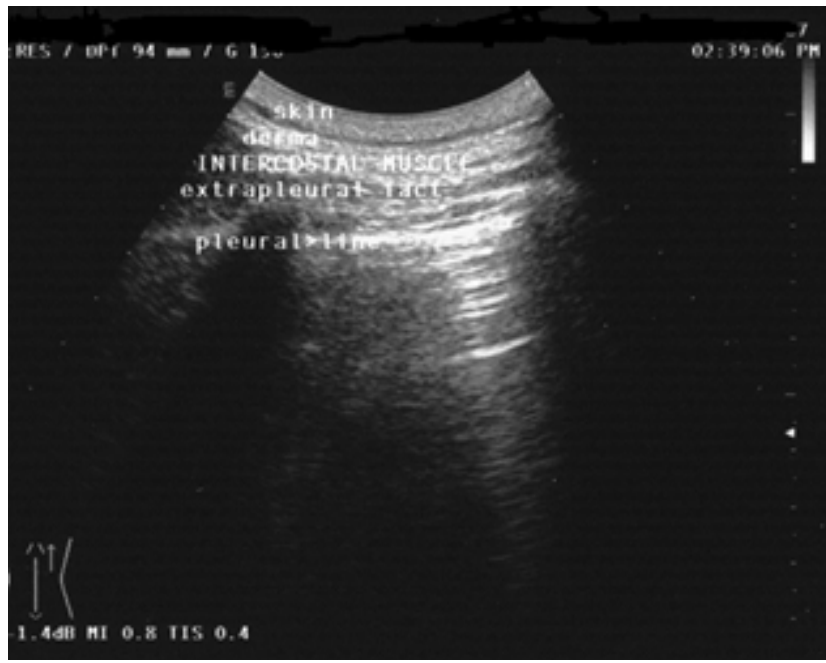

Figura 1. Estructuras que pueden explorarse mediante una ecografía torácica.

Fuente: (Targhetta, Bourgeois, Chavagneux, \& Balmes, 2002).

La pleura parietal está inmóvil, mientras que la pleura visceral se mueve durante la respiración ("signo de deslizamiento o deslizamiento"). En sujetos sanos, la marcada diferencia en los niveles de impedancia acústica de los tejidos blandos y las estructuras llenas de aire suele producir dos tipos de artefactos: el artefacto " cola de cometa ", que consiste en reverberaciones hiperecoicas paralelas que se extienden verticalmente desde la interfaz pleural hasta el borde opuesto de la pantalla, y los " artefactos de reverberación ", que son líneas hiperecoicas horizontales concéntricas que representa la interfaz entre la pleura y la pared torácica (Figura 2). 
debe confirmarse mediante toracocentesis exploratoria y biopsia. Según estudios, la presencia dentro del líquido de derrame de numerosas partículas flotantes ecogénicas que se arremolinan en respuesta a los movimientos respiratorios o cardíacos (el patrón de remolino) es un fuerte predictor de un derrame pleural maligno.

Los derrames localizados cerca de la pared pulmonar, que suelen estar asociados a formaciones pulmonares sólidas o neumonía, son fáciles de detectar. Algunos derrames se mueven libremente cuando cambia la posición del paciente; los derrames encapsulados son menos móviles. Un derrame pleural encapsulado se puede diferenciar fácilmente de un fibrotórax, que es paralelo a las costillas y no se mueve con los movimientos respiratorios. (Lichtenstein, Meziere, Biderman, \& Gepner, 2011)

La ecografía también se puede emplear para controlar de forma continua la posición de la aguja durante los procedimientos de toracocentesis de diagnóstico y terapéuticos. Es particularmente valioso en este entorno debido a su capacidad para detectar cantidades mínimas de líquido (menos de $10 \mathrm{ml}$ ) dentro de los recesos costofrénicos.

En todos los casos, la ecografía es una herramienta valiosa para guiar la biopsia pleural, que se asocia con una baja tasa de complicaciones. "Varias otras lesiones de la pared torácica también se pueden detectar con ecografía, incluidos quistes, abscesos, hematomas, tumores benignos y diversas lesiones que afectan las costillas (metástasis, osteomielitis bacteriana, lesiones tuberculosas)" (Targhetta R. , 2008). En presencia de afectación osteomielítica, las costillas presentan márgenes irregulares y suelen estar rodeadas de infiltrados anecoicos.

Los hallazgos que surgen de la ecografía torácica no siempre son específicos, pero esta modalidad de imagen puede ser útil para evaluar los cambios pleurales, por ejemplo, extensión, interrupción, desplaza- miento de la línea pleural ecoica, su inmovilidad durante los movimientos respiratorios. La biopsia de corte transtorácica guiada por ecografía también puede ser útil para diagnosticar lesiones en las regiones periféricas del tórax, que todavía se basa principalmente en radiografías de tórax, tomografía computarizada y resonancia magnética.

\section{Enfermedades pulmonares}

La ecografía también puede detectar la enfermedad pulmonar siempre que no haya aire entre la sonda y la lesión y el haz llegue a la pleura. Incluso una fina capa $(1-2 \mathrm{~cm})$ de aire puede reducir seriamente la visualización de lesiones sólidas, independientemente de su tamaño. "En ciertos casos, la ecografía también puede revelar lesiones pulmonares más profundas, por ejemplo, cuando el parénquima circundante está consolidado, es decir, atelectásico, o cuando la lesión está rodeada por un derrame pleural, que actúa como una ventana acústica" (El Fortia, El Gatit, \& Bendaoud, 2016).

Las lesiones pulmonares uniformemente anecoicas, generalmente bien delimitadas, pueden representar quistes (congénitos, broncogénicos, parasitarios o pleurales) o, más raramente, infartos pulmonares. Los lipomas, que son tumores benignos, aparecen en la ecografía como nódulos localizados que son hipoecoicos o anecoicos figura 4. La presencia de ecos dentro de un quiste anecoico hipo ecogénico es indicativa de una lesión 'compleja', como un absceso, un hematoma, una neoplasia necrótica o un quiste hidatídico multivesicular figura 5. "Los abscesos pulmonares aparecen como colecciones circunscritas de líquido corpuscular. Pueden detectarse fácilmente con ecografía cuando se encuentran en las regiones periféricas del pulmón, cerca de la pleura parietal o asociadas con adherencias pleurales" (Yang, Chang, \& Yu, 2002). 


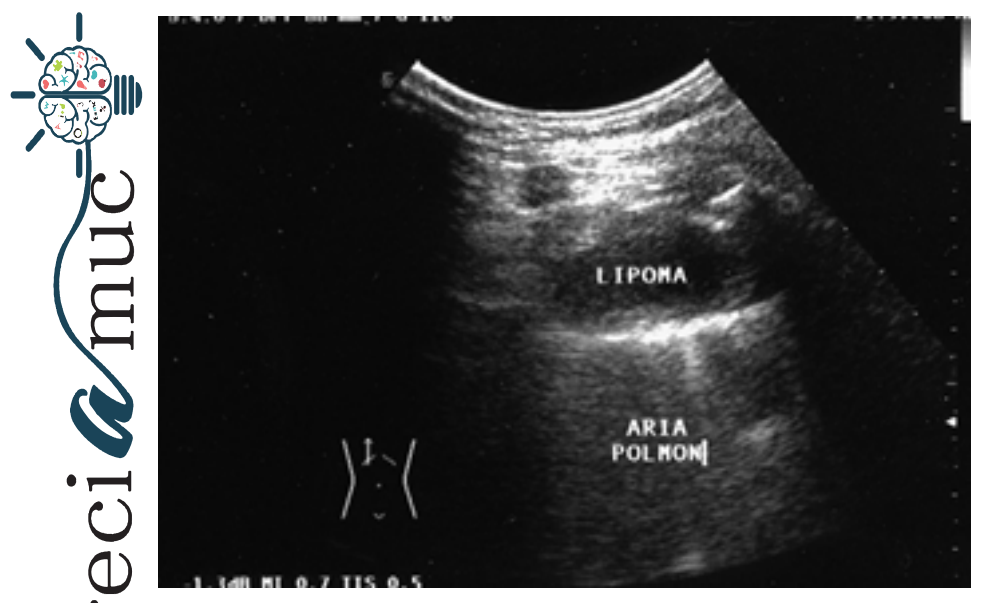

Figura 4. El lipoma pulmonar aparece en la ecografía como un nódulo hipoanecoico localizado.

Fuente: (Yang, Luh, \& Chang, 2012).

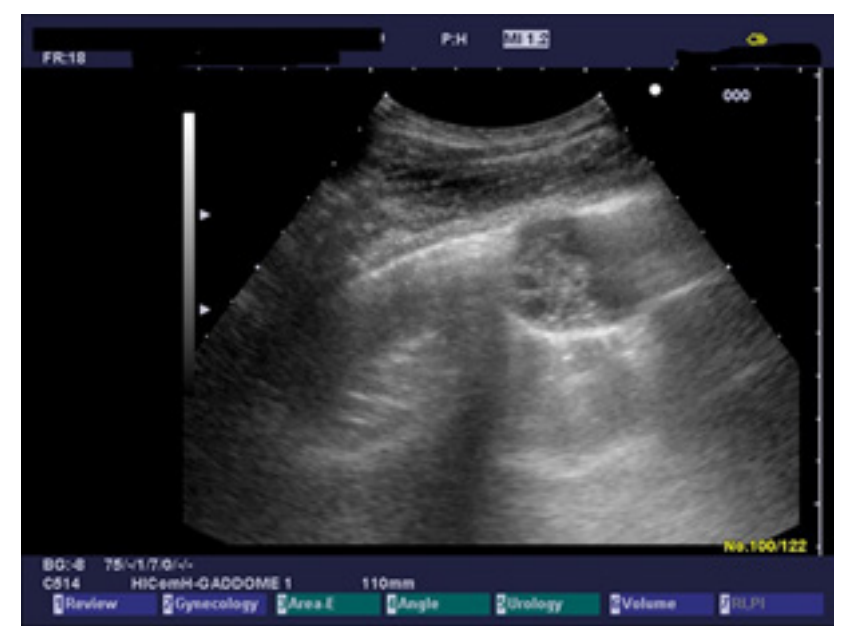

Figura 5. Quiste equinocócico pulmonar con pequeños quistes hijos (tipo CE3b en la clasificación de la OMS).

Fuente: (Yang, Luh, \& Chang, 2012).

Las neumoconiosis, un grupo de enfermedades pulmonares causadas por partículas inhaladas, no están asociadas con los patrones típicos de la ecografía, aunque comúnmente se detecta un engrosamiento pleural irregular en las fases avanzadas de la enfermedad. La ubicación de la lesión en el pulmón "puede ayudar en el diagnóstico: la presencia de nódulos subpleurales o de lesiones en el vértice dorsal del pulmón sugieren silicosis, mientras que las lesiones periféricas en los lóbulos inferiores son un rasgo característico de asbestosis" (Yang, Luh, \& Wu, Lung, 2000).

De acuerdo con (Sperandeo, Sperandeo, \& Varriale, 2016), "la ecografía puede detectar la enfermedad pulmonar intersticial si el intersticio del pulmón periférico está afectado". Los hallazgos típicos de ecografía asociados con la fibrosis pulmonar incluyen:

1-fragmentación y engrosamiento irregular de la línea pleural, especialmente en los lóbulos inferiores (hallazgos que son independientes de la gravedad de la enfermedad) (Figura 11);

2-atenuación del signo de deslizamiento fisiológico, que está relacionado con el estadio de la enfermedad;

3-inmovilidad del diafragma, que se visualiza mejor en una exploración del tórax derecho realizada cuando el paciente está en posición supina (enfermedad en etapa terminal);

4-artefactos de reverberación múltiple (etapa avanzada).

Todas estas anomalías se pueden detectar en ambos pulmones, lo que es un reflejo de la naturaleza difusa del proceso fibrótico.

Los hallazgos de la ecografía asociados con el fibrotórax son una línea pleural engrosada asociada con un signo de deslizamiento atenuado. La sarcoidosis pulmonar se asocia con micronodulación, relacionada con el grosor del tejido conectivo en los lóbulos superiores del pulmón. En algunos casos, también se observan pequeños derrames pleurales en las bases pulmonares. (Sperandeo, Varriale, \& Filabozzi, 2007)

La atelectasia pulmonar se diagnostica fácilmente mediante ecografía. "Se pueden distinguir dos formas; una forma central causada por la obstrucción de las vías respiratorias y una forma periférica relacionada con la compresión del parénquima por derrame de líquido, aire (neumotórax), traumatismo, tejido fibroso difuso o tumores malig- 
nos" (Kirkpatrick, Ng, \& Dulchavsky, 2011).

Los procesos inflamatorios, como la neumonía o la consolidación pulmonar, que afectan las regiones periféricas del pulmón también pueden detectarse mediante ecografía. El tejido pulmonar inflamado suele aparecer como una "lesión hipoecoica o anecoica o, en ocasiones, como una imagen hipoehiperecoica con márgenes borrosos. Dentro de los bordes de la lesión hay líneas hiperecoicas correspondientes a bronquios llenos de aire (el broncograma aéreo ecográfico)" (Kirkpatrick, Ng, \& Dulchavsky, 2011)

Las enfermedades inflamatorias del pulmón se asocian con frecuencia con derrames pleurales basales, que pueden detectarse mediante ecografía. "Esta modalidad también puede ser útil en el seguimiento de un paciente con consolidación pulmonar, ya que permite visualizar los cambios que involucran el proceso inflamatorio después de la terapia" (Kirkpatrick, Ng, \& Dulchavsky, 2011).

Desde entonces, se ha utilizado regularmente la ecografía solo para descartar la posibilidad de neumotórax después de una toracocentesis o una biopsia pleuropulmonar. La precisión diagnóstica de la ecografía en la mayoría de los casos de neumotórax e hidroneumotórax parece superior a la de la radiografía de tórax convencional. Salvo en unos pocos casos dudosos, este último examen ya no es obligatorio después de procedimientos terapéuticos o diagnósticos invasivos. Sin embargo, "en presencia de fibrotórax difuso, el diagnóstico de neumotórax mediante ecografía es muy difícil porque el signo de deslizamiento no es fácilmente detectable en estos pacientes" (Targhetta, Bourgeois, Chavagneux, \& Balmes, 2002).

\section{Procedimientos invasivos pleurales y pulmonares}

La ecografía torácica se utiliza ampliamente para guiar la colocación de la aguja durante los procedimientos de toracocentesis, lo que reduce el riesgo de neumotórax. "Según se informa, el neumotórax se produce en el 7 al 15\% de los pacientes que se someten a una toracocentesis a ciegas, pero la frecuencia desciende al 0,5\% cuando se utiliza la guía con aguja de ultrasonido" (Liao, Chen, \& Chang, 2000).

Como se mencionó anteriormente, la ecografía puede proporcionar información sobre las características del derrame (simple, encapsulado u organizado), lo cual es importante para definir la naturaleza de las opacidades que se observan en las radiografías de tórax (por ejemplo, diferenciación del derrame pleural y la consolidación pulmonar). Cada departamento de emergencias debe estar equipado con un escáner de ecografía, ya que, puede desempeñar un papel clave en la verificación de la sospecha clínica de derrame pleural masivo en pacientes con dificultad respiratoria aguda. También permite un drenaje rápido del tórax con una tasa baja de neumotórax. "En pacientes críticamente enfermos, incluso los derrames masivos a menudo se pueden drenar en un tiempo razonablemente corto con un aspirador de bajo flujo y baja presión con una aguja de pequeño calibre (20 G)" (Liao, Chen, \& Chang, 2000).

Las lesiones pleurales que pueden evaluarse con biopsia transtorácica guiada por ecografía incluyen engrosamiento nodular, que aparece como micro nodulación hipoecoica a menudo combinada con engrosamiento de la línea pleural; y mesoteliomas pleurales, que aparecen como lesiones mixtas hiperecoicas, a menudo de formas múltiples, que se asocian con derrames serohemáticos. (Las lesiones pleurales benignas, como fibromas o lipomas, son raras).

En casos de neumonía, a menudo es imposible determinar la etiología basándose en la evaluación bacteriológica del esputo y / o líquido del derrame pleural, hemocultivos y examen serológico de muestras de orina. Las tasas de complicación comunicadas son bajas (neumotórax en el 1-2\% de 
los casos). En casos de empiema pleural o absceso pulmonar periférico, la guía ecográfica es una herramienta clínica fundamental (Zimmer, Rost, \& Patan, 2007).

La ecografía es capaz de detectar tumores pulmonares sólo cuando se localizan en las regiones periféricas del órgano. Actualmente utilizamos agujas de aspiración y corte 20e21 G (Histo-cut, Sterylab, Rho, Italia o Biomol Hospital Service, Aprilia, Italia).

Los tumores de pulmón diagnosticados con mayor frecuencia por punción-aspiración guiada por ecografía son adenocarcinomas $(41 \%)$, carcinomas de células escamosas (37\%), carcinomas anaplásicos de células grandes (17\%), microcitomas o metástasis (5\%). Las tasas de exactitud diagnóstica comunicadas en la bibliografía para la biopsia por aspiración con aguja transtorácica guiada por ecografía oscilan entre el 85\% y el 96\% (Dupuy, Zagoria, \& Akerley, 2000).

La aplicación más reciente de la ecografía en el estudio de las enfermedades pleurales y pulmonares es la ecografía endoluminal. Esta compleja técnica, que actualmente se realiza sólo en unos pocos centros altamente especializados, incluye (1) ecoendoscopia transesofágica, que puede revelar anomalías (adenopatías y masas neoplásicas) entre la aorta y las venas pulmonares centrales, eliminando la necesidad de biopsia; (2) ecografía Endo bronquial, que se puede utilizar para visualizar lesiones intra y subparietales de los bronquios, así como la extensión peribronquial de una masa bronquial, y para caracterizar el patrón vascular de estas lesiones; (3) Ecografía durante la toracoscopia, que puede identificar nódulos diminutos que son imposibles de detectar con otras herramientas.

\section{Conclusiones}

Es evidente que la ecografía torácica está adquiriendo una importancia cada vez mayor en el estudio diagnóstico de la patología pleural y, en los últimos años, varios estudios han abordado su utilidad y ventajas en este ámbito. Además de la falta de exposición a la radiación, parece ser tan eficaz como la radiografía de tórax para detectar o excluir el neumotórax, es superior a la radiografía de tórax para detectar y caracterizar el derrame pleural y se considera el método de elección para guiar la aspiración de líquido pleural y biopsia percutánea de lesiones de base pleural.

A diferencia de los métodos radiológicos, los dispositivos de ultrasonido portátiles permiten la exploración en casi cualquier lugar, y tal ventaja puede jugar un papel importante en los servicios de urgencias y unidades de cuidados intensivos; sin embargo, a pesar de estas ventajas, tiene algunos límites y no puede considerarse como una alternativa a la TC torácica para el estudio de la patología pleural.

La ecografía solo logra una mala visualización del mediastino (en particular, el mediastino posterior) y las ondas ecográficas se ven obstaculizadas por el aire y las estructuras óseas. Como consecuencia, no proporciona ninguna información diagnóstica en presencia de enfisema subcutáneo y no puede visualizar lesiones subescapulares, paravertebrales y retroesternales. Aunque puede proporcionar información útil y confiable sobre la infiltración pleural parietal de los tumores pulmonares, la CT es superior a la ecografía para investigar enfermedades pleurales focales y difusas, ya que permite evaluar todas las partes de la pleura, incluida la pleura mediastinal y puede delinear mejor los componentes pulmonar y pleural de una masa.

Otra de las limitaciones es que la ecografía torácica depende estrictamente del operador y solo los examinadores capacitados con mucha experiencia pueden obtener resultados buenos y confiables. No obstante, puede proporcionar información diagnóstica inmediata en situaciones particulares, como la sospecha de derrame pleural, la detección o exclusión de neumonía, neumotórax y después de procedimientos in- 
tervencionistas o en pacientes críticamente enfermos e inestables. Además, puede aportar información interesante en casi todas las enfermedades pleurales más frecuentes por tal razón, debería incluirse como una herramienta útil y complementaria en un abordaje multimodal actualizado del trabajo diagnóstico de patología pleural.

\section{Bibliografía}

Alcazar, B., \& Arnedillo, A. (2016). Aplicaciones de la ecografía en neumología. Revista Española de Patología Torácica , 1, 1-79.

Bokor, D., Chambers, J., \& Rees, P. (2011). Clinical safety of SonoVue, a new contrast agent for ultrasound imaging, in healthy volunteers and in patients with chronic obstructive pulmonary disease. Invest Radiol , 104-9.

Bolívar, J. (2015). Investigación Documental. México. Pax.

Castro, J. (2016). Técnicas Documentales. México. Limusa.

Chian, C., Su, W., \& Soh, L. (2014). Echogenic swirling pattern as a predictor of malignant pleural effusions in patients with malignancies. Chest, 129-34.

Davila, A. (2015). Diccionario de Términos Científicos. . Caracas: Editorial Oasis.

Dupuy, D., Zagoria, R., \& Akerley, W. (2000). Percutaneous radiofrequency ablation of malignancies in the lung. AJR Am J Roentgenol , 57-9.

El Fortia, M., El Gatit, A., \& Bendaoud, M. (2016). Ultrasound wall-sign in pulmonary echinococcosis (new application). Ultraschall Med , 553-7.

Kirkpatrick, A., Ng, A., \& Dulchavsky, S. (2011). Sonographic diagnosis of a pneumothorax inapparent on plain radiography: confirmation by computed tomography. J Trauma, 750-2.
Liao, W., Chen, M., \& Chang, Y. (2000). US-guided transthoracic cutting biopsy for peripheral thoracic lesions less than $3 \mathrm{~cm}$ in diameter. Radiology 685-91.

Lichtenstein, D., Meziere, G., Biderman, P., \& Gepner, A. (2011). The comet-tail artifact: an ultrasound sign ruling out pneumothorax. Intensive Care Med , 347.

Sperandeo, M., Caturelli, E., Sperandeo, G., \& Camagna, A. (2012). New technique of thoracentesis in massive hydrothorax. J Hepatol , 209.

Sperandeo, M., Sperandeo, G., \& Varriale, A. (2016). Contrast-enhanced ultrasound (CEUS) for the study of peripheral lung lesions: a preliminary study. Ultrasound Med Biol , 1467-72.

Sperandeo, M., Varriale, A., \& Filabozzi, P. (2007). The role of thoracic echography in the pleuro-pulmonary disease: ambitious or standard project? Intern Emerg Med , 204.

Targhetta, R. (2008). Sonographic approach to pulmonary disease. JEMU , 217-21.

Targhetta, R., Bourgeois, J., Chavagneux, R., \& Balmes, P. (2002). Diagnosis of pneumothorax by ultrasound immediately after ultrasonically guided aspiration biopsy. Chest, 855-6.

Yang, P., Chang, D., \& Yu, C. (2002). Ultrasound-guided core biopsy of thoracic tumors. Am Rev Respir Dis, 763-7.

Yang, P., Luh, K., \& Chang, D. (2012). Value of sonography in determining the nature of pleural effusion: analysis of 320 cases. AJR Am J Roentgenol ,29-33.

Yang, P., Luh, K., \& Wu, H. (2000). Lung tumors associated with obstructive pneumonitis: US studies. Radiology , 717-20.

Zimmer, T., Rost, T., \& Patan, M. (2007). Endoscopic ultrasound of pathological mediastinal findings. Radiologe , 37.

\section{CITAR ESTE ARTICULO:}

Sánchez Riera, E. E., Osorio Vera, L. E., Quisiguiña Avellan, L. E., \& Herrera Vera, M. X. (2021). Utilidad de la ecografía torácica en pacientes con enfermedad pleuropulmonar. RECIAMUC, 5(1), 257-367. https://doi.org/10.26820/ reciamuc/5.(1).ene.2021.357-367

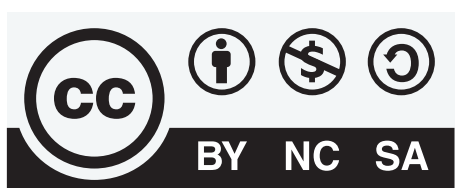

CREATIVE COMMONS RECONOCIMIENTO-NOCOMERCIAL-COMPARTIRIGUAL 4.0. 